\title{
Regio- and diastereoselective synthesis of trans-dihydrofuran-3- carboxamides by radical addition of 1,3-dicarbonyl compounds to acrylamides using manganese(III) acetate and determination of exact configuration by $X$-ray crystallography
}

\author{
Mehmet Yilmaz, ${ }^{a^{*}}$ Ash Ustalar, ${ }^{\text {a }}$ Bedriye Uçan, ${ }^{\text {b }}$ and A. Tarık Pekel ${ }^{\text {b }}$ \\ ${ }^{1}$ Department of Chemistry, Faculty of Arts and Sciences, Kocaeli University, 41380 \\ Umuttepe, Kocaeli, Turkey \\ ${ }^{2}$ Department of Chemistry, Faculty of Science, Ankara University, 06100 Tandogan, Ankara, \\ Turkey \\ E-mail: mehmet.yilmaz@kocaeli.edu.tr
}

DOI: https://doi.org/10.24820/ark.5550190.p009.855

\begin{abstract}
In this study, we investigated the radical addition of 1,3-dicarbonyl compounds to acrylamide derivatives including phenyl, 2-thienyl and 5-methyl-2-furyl groups mediated by manganese(III) acetate. trans-3-Carboxamide-dihydrofurans were obtained in modarate to good yields, as well as regio- and diastereoselectievly. Structural analyses of these compounds were made by NMR techniques such as HMBC and NOSY spectra. Also, exact configuration and structures of these $(\mathbf{7 b}, \mathbf{7} \mathbf{i}$ and $\mathbf{7 j}$ ) compounds were determined by X-ray crystallography.
\end{abstract}

Keywords: Manganese(III) acetate, radical addition, cyclization, dihydrofuran-carboxamide, Xray analysis, diastereoselective

\section{Introduction}

Substituted dihydrofurans constantly occur as subunits for many medicinally important compounds and biologically active natural compounds, such as clerodin, aflatoxin $\mathrm{B}_{1}$, rocoglamide, austocystin $\mathrm{A}$ and fercoprolone. ${ }^{1-3}$ On the other hand, the carboxamide group is an important structural moiety in many biologically active compounds and in some drugs, such as acridine carboxamide (topoisomerase inhibitor), nicotineamide, pyrazineamide (a drug for tuberclosis), dacarbazine (antineoplastic), valpromide (a drug for epilepsy). It was reported that some dihydrofurans including carboxamide, ${ }^{4}$ and carbonitrile ${ }^{5}$ show antifungal and antibacterial activity. Thus, development of effective synthetic methods to prepare polysubstituted dihydrofurans has been focused on this area. 
Manganese(III) acetate ${ }^{6-10}$ and cerium(IV) ammonium nitrate (CAN) $)^{11-14}$ are widely used for $\mathrm{C}-\mathrm{C}$ bond formation and synthesis of polyfunctional organic compounds. It is well known that $\mathrm{Mn}(\mathrm{OAc})_{3}$ or $\mathrm{CAN}$ mediated radical reaction of 1,3-dicarbonyl compounds with alkenes produce dihydrofuran derivatives. $\mathrm{Mn}(\mathrm{OAc})_{3}, \mathrm{CAN}$ or $\mathrm{Ag}(\mathrm{I}) /$ Celite mediated radical reactions of 1,3dicarbonyl compounds with unsymmetrically alkenes such as (1-pyridin-2-yl)-enones, ${ }^{9}$ unsaturated ketones, ${ }^{15,16}$ cinnamates, ${ }^{7,14}$ and unsaturated ether, ${ }^{11,}{ }^{17}$ have been reported. Our research group has focused on the radical reaction of 1,3-dicarbonyls and 3-oxopropanenitriles with unsaturated systems. ${ }^{18-27}$ We first reported the reaction of $(E)$-2-styrylthiophene and $(E)$-2methyl-5-styrylfuran with 3-oxopropanenitriles, ${ }^{18,} 20$ fluorinated-1,3-dicarbonyls ${ }^{21}$ and 1,3diketones $^{22,23}$ lead to formation of 2-thienyl and 2-furylsubstituted trans-dihydrofurans (1 and 2). Although there are two possible different cyclization routes with these reactions, we reported that, this cyclization occurs only at the $\mathrm{C}-1$ carbon (adjacent to thienyl or furyl groups) regioselectively (Figure 1). ${ }^{18,20}$
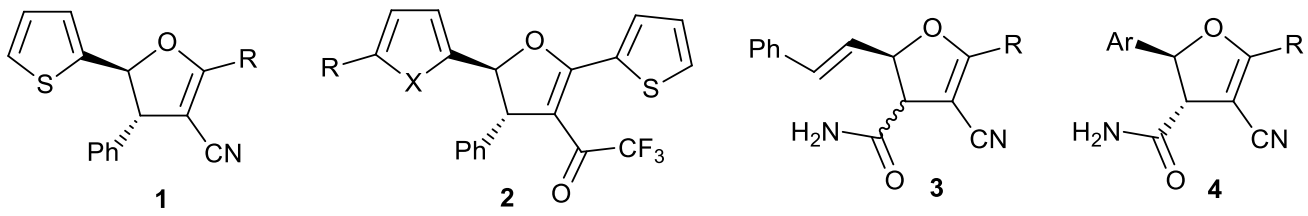

Figure 1

Previously, we obtained cis- and trans-diastereomer mixtures of dihydrofurans (3) from the reaction of (E,E)-5-phenyl-2,4-pentandienamide with 3-oxopropanenitrile. We clearly reported that coupling constants of the $\mathrm{H} 2$ and $\mathrm{H} 3$ vicinal protons of this compounds are $J_{\text {cis }} 9.6-10.3 \mathrm{~Hz}$ and $J_{\text {trans }} 6.8-7.5 \mathrm{~Hz}$, respectively. ${ }^{19,} 27$ Based on the coupling constants of the transdiastereomers, we decided that 2,3-dihydrofuran-3-carboxamides (4) obtained from the reaction of 3-oxopropanenitriles with (2E)-3-(2-thienyl)acrylamide and (2E)-3-(5-methyl-2furyl)acrylamide are in trans configuration (Fig 1). ${ }^{19}$

Depending on the substituents attached to dihydrofuran ring, coupling constant of the vicinal protons can show some inequality. Hence, if both cis- and trans-diastereomers do not form in the reaction of unsymmetrical alkenes, coupling constants of the vicinal protons or NOSY (or NOEDIFF) spectra may not be inadequate to determine configuration of the dihydrofurans in all cases. The most exact proof of structure and configuration of the compounds can only be obtained from X-ray crystallography. Thus, in the present study, we reported the radical addition of acrylamide derivatives with various 1,3-dicarbonyl compounds using $\mathrm{Mn}(\mathrm{OAc})_{3}$, lead to trans-3-carboxamide-substituted dihydrofurans as regio- and diastereoselectively. Absolute structure and configuration of these compounds were determined by X-ray analysis. 


\section{Results and Discussion}

(2E)-3-Phenylacrylamide $\quad 5 a, \quad(2 E)-3-(2-t h i e n y l) a c r y l a m i d e \quad 5 b, \quad(2 E)-3-(5-m e t h y l-2-$ furyl)acrylamide $\mathbf{5 c}$ and (2E)-3-(3-thienyl)acrylamide $\mathbf{5 d}$ were prepared by the reaction of suitable acyl chloride and $\mathrm{NH}_{3} / \mathrm{NaOH}$ as described in literature. ${ }^{28,} 19$

Table 1. The reaction of 1,3-dicarbonyls (6a-d) with (2E)-3-phenylacrylamide 5a.

Entry 1,3-dicarbonyl acrylamide dihydrofuran

${ }^{A}$ Yield of isolated product based on the conjugated amides 5a.

Treatments of (2E)-3-phenylacrylamide 5a with dimedone $\mathbf{6 a}$ and 1,3-cyclohexandione $\mathbf{6 b}$ in the presence of $\mathrm{Mn}(\mathrm{OAc})_{3}$ in $\mathrm{HOAc}$ in a 2:1:3 molar ratio (1,3-dicarbonyl : acrylamide : $\left.\mathrm{Mn}(\mathrm{OAc})_{3}\right)$ at $70{ }^{\circ} \mathrm{C}$ gave 2-phenyl-3-carboxamide-dihydrobenzofurans $7 \mathbf{a}$ in $68 \%$ and $\mathbf{7 b}$ in $55 \%$ yields (Table 1). In the ${ }^{1} \mathrm{H}$ NMR spectra of these compounds, the $\mathrm{H}-2$ and $\mathrm{H}-3$ (C8-H and $\mathrm{C} 7-\mathrm{H}$ in the $\mathrm{x}$-ray graphic for $\mathbf{7 b}$, respectively) protons of the dihydrobenzofurans $\mathbf{7 a}$ and $\mathbf{7 b}$ appeared as two doublets with a vicinal coupling constant of $J 6.0 \mathrm{~Hz}$. The H-2 protons $(\delta 6.14$ and $6.27 \mathrm{ppm}$ ) resonate at low field due to inductive effect of neighbouring etheric oxygen atom. Since the HMBC spectra of 7a shows that the H-2 proton correlates with the ortho-carbon of phenyl resonates at $125.3 \mathrm{ppm}$, phenyl group attached to the C-2 carbon in the dihydrobenzofuran ring. Also, we verified absolute configuration and the assignment of regioand diastereoselectivity through X-ray analysis of $7 \mathbf{b}(2 R, 3 R)$ in Figure 2. 
Treatments of ethyl 3-oxobutanoate $\mathbf{6 c}$ and 2,4-pentanedione $\mathbf{6 d}$ with 5a gave trans-2,3dihydrofurans $\mathbf{7 c}$ and $\mathbf{7 d}$ in moderate yields, respectively. Compounds $\mathbf{7 c}$ and $\mathbf{7 d}$ show only two doublets for two protons at C2- and C3-position with the coupling constants of 5.2 and $4.8 \mathrm{~Hz}$, which obviously indicated that these 2,3-dihydrofurans are in trans-configuration.

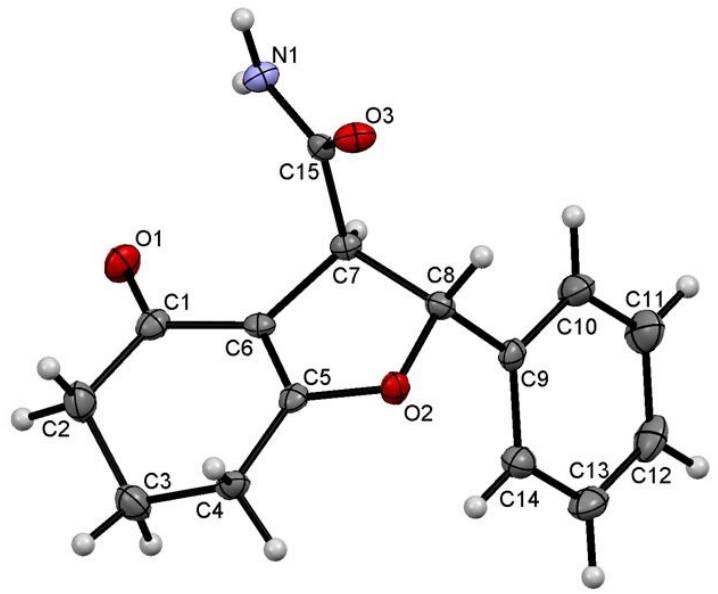

Figure 2. Molecular structure of the compound $\mathbf{7 b}$.

It has been reported that the vicinal coupling constants of two methine protons in cisdihydrofuran are $J_{\text {cis }} 8-11 \mathrm{~Hz}$, while in trans-dihydrofurans vicinal coupling constants are $J_{\text {trans }}$ 2.5-7.5 Hz. ${ }^{18-27,} 29,30$ Based on this information, the previously assigned cis-stereomer should likely be the trans-stereomer for the dihydrofurans obtained from the $\mathrm{Mn}(\mathrm{OAc})_{3}$ mediated radical reactions of 1,3-dicarbonyl compounds with chalcones, ${ }^{15} 2$-styrylthiophene ${ }^{10}$ and from the radical reactions of 1,3-dicarbonyls with cinnamates, ${ }^{14}$ unsaturated ketones ${ }^{16}$ promoted by CAN as these dihydrofurans had vicinal coupling constants of 5.8-7.2 Hz.

As seen in the Table 2, radical addition of dimedone $\mathbf{6 a}$ to $\mathbf{5 b}$ afforded trans-3-(2-thienyl)2,3-dihydrobenzofuran-3-carboxamide $7 \mathrm{e}$ which was characterized by ${ }^{1} \mathrm{H} \mathrm{NMR},{ }^{13} \mathrm{C}$ NMR, NOSY and HMBC spectra. In the ${ }^{1} \mathrm{H}$ NMR spectrum of 7e, vicinal coupling constants of $\mathrm{H}-2$ and $\mathrm{H}-3$ are $J_{\text {trans }} 5.6 \mathrm{~Hz}$. In the HMBC spectrum, H-3 proton resonated at $4.1 \mathrm{ppm}$ correlates with the thiophene carbon resonated at $142.5 \mathrm{ppm}$. These results show that 2-thienyl attached to the C-2. Moreover, similar results were obtained by the radical reaction of 1,3-dicarbonyls $\mathbf{6 b - d}$ with $\mathbf{5 b}$ forming trans-3-(2-thienyl)-dihydrofuran-3-carboxamides $\mathbf{7 f - h}$ with vicinal coupling constants of $5.2-4.2 \mathrm{~Hz}$ appearing in corresponding ${ }^{1} \mathrm{H}$ NMR spectra (Table 2, Entries 2-4). On the other hand, trans-3-(5-methyl-2-furyl)dihydrobenzofuran-3-carboxamide 7i was obtained by the treatment of dimedone 6a with (2E)-3-(5-methyl-2-furyl)acrylamide 5c in $80 \%$ yield. Reaction of $\mathbf{6 c}$ and $\mathbf{6 d}$ with $\mathbf{5 c}$ afforded trans-2,3-dihydrofuran-3-carboxamides $\mathbf{7 j}(67 \%)$ and $\mathbf{7 k}$ $(70 \%)$ in good yields (Table 2, Entries 6 and 7). Also, the radical reaction of (2E)-3-(3thienyl)acrylamide $\mathbf{5 d}$ with $\mathbf{6 a}$ gave corresponding compound $7 \mathbf{l}$ in $36 \%$ yield. All dihydrofurans were obtained in moderate to good yields. 
Related unsaturated amides used in radical reactions regained with column chromatography from each crude products in 15-25\% yields. To accurately understand if there are any possible compounds (especially cis- isomers) formed in this type of radical addition reactions, the crude products from experiments 7a and 7e were analysed with LC-MS (without purification). The results from these analyses showed that there are no isomers with same masses formed beside the trans- products.

Table 2. Radical reaction of 1,3-dicarbonyl compounds 7a-d with $\mathbf{2 c}$ and $\mathbf{2 d}$

entry

A: Yield of isolated product based on the conjugated amides $\mathbf{5 b}$ and $\mathbf{5 c}$. Moreover, structural assignment of compounds $7 \mathbf{i}(2 S, 3 S)$ and $7 \mathbf{j}(2 R, 3 R)$ were further characterized by $\mathrm{x}$-ray single 
crystal analysis (Figs 3 and 4), as a result of this, we can conclude that these vicinal protons are definitely in trans-configuration.

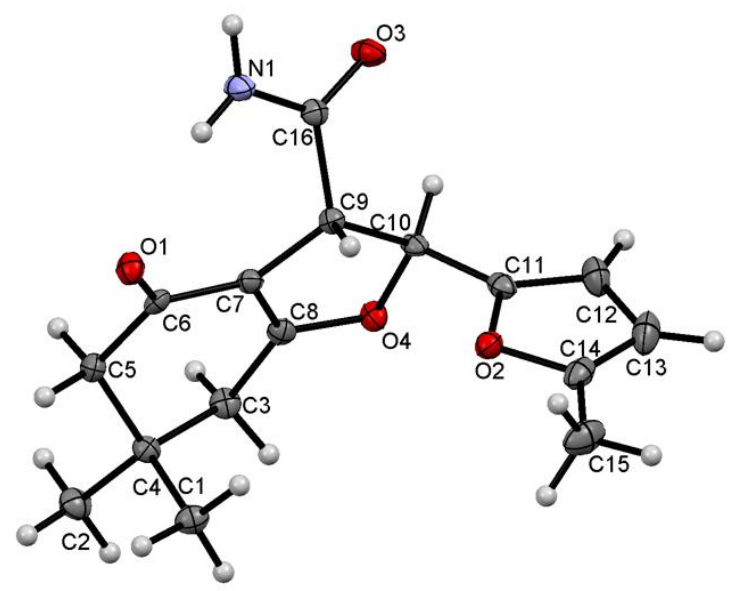

Figure 3. Molecular structure of the compound $7 \mathbf{i}$.

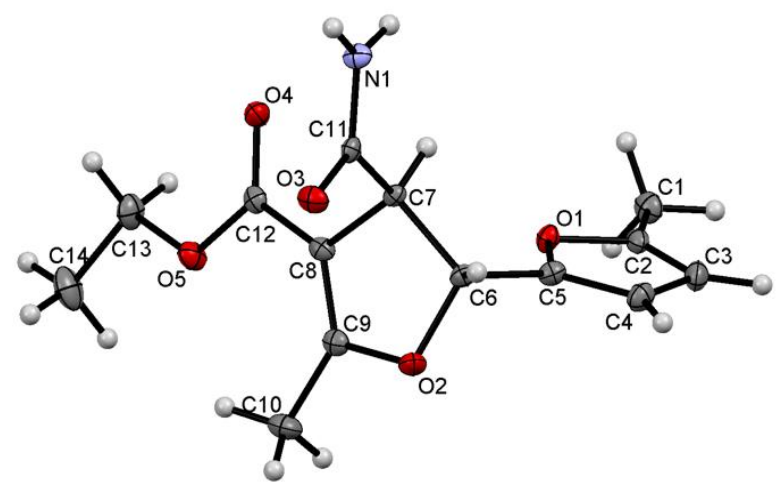

Figure 4. Molecular structure of the compound 7j. Displacement ellipsoids are drawn at the 50 $\%$ probability level. $\mathrm{H}$-atoms are shown as small spheres of arbitrary radii.

The proposed mechanism for the formation of dihydrofuran-carboxamides is illustrated in scheme 1. According to the mechanism, whilst $\mathrm{Mn}^{+3}$ reduces to $\mathrm{Mn}^{+2}$, a carbon radical forms on the 1,3-dicarbonyl. The intermediate product $\mathbf{A}$ can be added to acrylamides with two ways. An adduct intermediate $\mathbf{B}$ forms with pathway- $i$ then structure $\mathbf{B}$ is oxidized to carbocation $\mathbf{C}$ with $\mathrm{Mn}(\mathrm{OAc})_{3}$ and then enolizes to intermediate $\mathbf{E}$. Dihydrofuran $\mathbf{G}$ is formed by intramolecular cyclization of $\mathbf{F}$. Dihydrofuran $\mathbf{I}$ which occurred by the cyclization of the other adduct intermediate $\mathbf{H}$ obtained by pathway-ii. However, dihydrofuran I was not isolated, only dihydrofurans $\mathbf{G}$ were obtained as sole products. The reason behind regioselective addition is that intermediate $\mathbf{B}$ is being more stable than intermediate $\mathbf{H}$. Also, diastereoselectivity can be explained by intramolecular cyclization in structure $\mathbf{F}$ preferring trans-isomer which has less steric hindrance and more thermodynamically stable. 


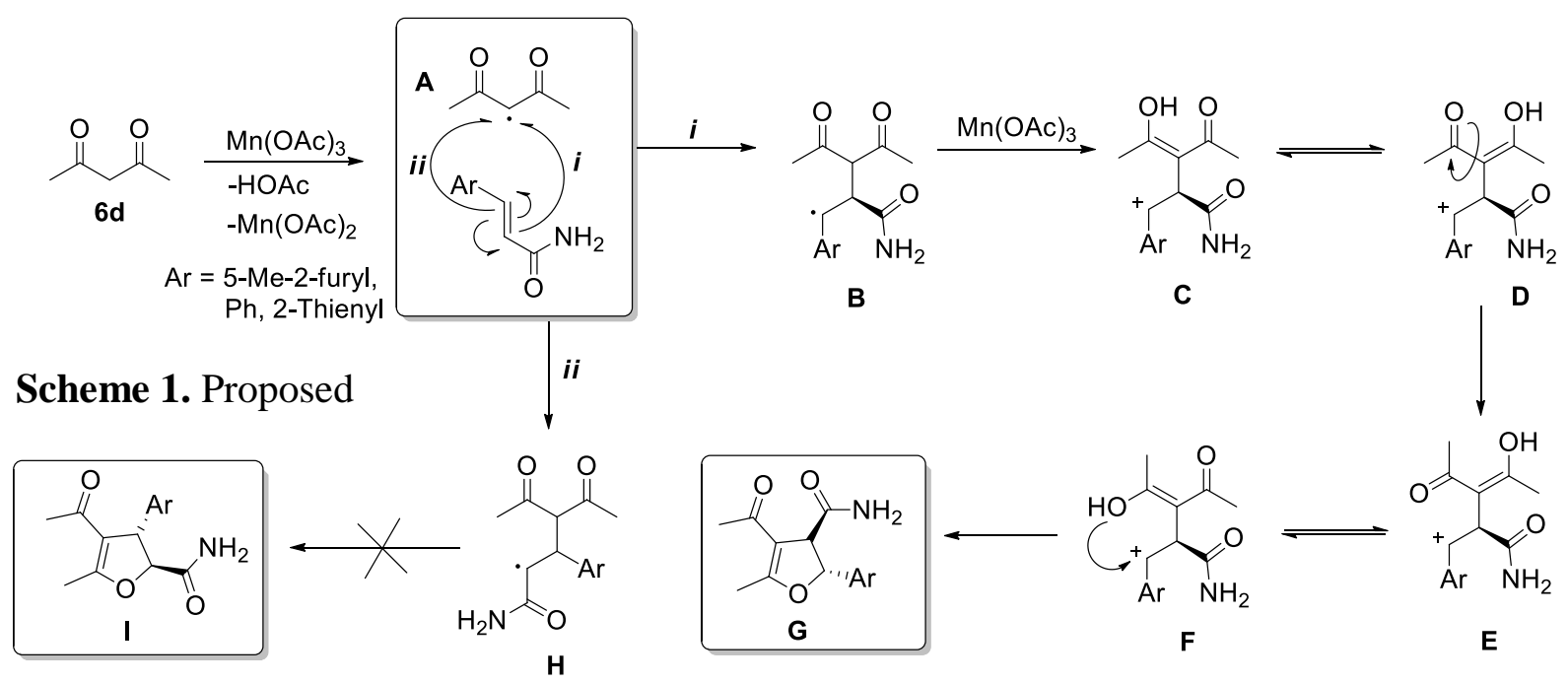

mechanism for the formation of trans-dihydrofuran-carboxamides.

\section{Conclusions}

We obtained 3-carboxamide-dihydrofurans in good yields as diastereoselectively by the manganese(III) acetate mediated reaction of acrylamide derivatives and 1,3-dicarbonyl compounds. It is seen that coupling constants of vicinal protons of the dihydrofurans in this work are 1-1.5 Hz less than that of 3-cyano-carboxamides. These results show that the substituents attached on the $\mathrm{C}=\mathrm{C}$ double bond in the dihydrofuran rings significantly effect on the coupling constants of vicinal protons. Our studies based on the X-ray analyses continue to determine substituent effects on the coupling constant of vicinal protons and definite configurations of some dihydofurans whose structure is open to debate.

\section{Experimental Section}

General. Melting points were determined on an electrothermal capillary melting point apparatus. IR spectra (ATR) were obtained with a Bruker Tensor-27 400-4000 cm-1 range with $2 \mathrm{~cm}^{-1}$ resolution. ${ }^{1} \mathrm{H}$ NMR, ${ }^{13} \mathrm{C}$ NMR, HMBC, NOSY, spectra were recorded on a Bruker Avance DPX-400 MHz and Varian Mercury-400 High performance Digital FT-NMR spectrophotometers. High Resolution Mass Time-of-Flight spectra (TOF) were measured on a Agilent 1200/6210 LC/MS spectrophotometer. Unit cell measurements and intensity data collection was performed on an Bruker APEX II QUAZAR three-circle diffractometer using monochromatized Mo K $\alpha$ microfocus sealed-tube $(\lambda=0.71073 \AA)$ using $\varphi$ and $\omega$ technique at $120 \mathrm{~K}$. Thin layer chromatography (TLC) was performed on Merck aluminum-packed silica gel 
plates. Purification of the products was performed by column chromatography on silica gel (Merck silica gel 60, 40-63 mm) or preparative TLC on silica gel of Merck ( $\mathrm{PF}_{254-366 \mathrm{~nm}}$ ).

General procedure for dihydrofuran-carboxamides (7). A solution of manganese(III) acetate dihydrate $(3 \mathrm{mmol}, 0.804 \mathrm{~g})$ in $10 \mathrm{~mL}$ of glacial acetic acid was heated on oil bath under a nitrogen atmosphere at $80{ }^{\circ} \mathrm{C}$ until it dissolved. After $\mathrm{Mn}(\mathrm{OAc})_{3}$ dissolved completely, the solution was cooled down to $60{ }^{\circ} \mathrm{C}$. A solution of 1,3-dicarbonyl compound (2 mmol) and acrylamide $(1 \mathrm{mmol})$ in $5 \mathrm{~mL}$ of acetic acid was added to this mixture and the temperature was raised to $70{ }^{\circ} \mathrm{C}$. The reaction was complete when the dark brown color of the solution disappeared (monitoring by TLC). $30 \mathrm{~mL}$ water was added to the solution and extracted by $\mathrm{CHCl}_{3}(3 \times 20 \mathrm{~mL})$. The combined organic extracts were neutralized with $10 \mathrm{~mL}$ of satd $\mathrm{NaHCO}_{3}$ solution, and dried over anhydrous $\mathrm{Na}_{2} \mathrm{SO}_{4}$ and evaporated. The crude products were purified by column chromatography or preparative TLC $(20 \times 20 \mathrm{~cm}$ plates, $2 \mathrm{~mm}$ thickness $)$ using $\mathrm{n}$ hexane/EtOAc $(1: 1)$ as eluent.

trans-6,6-Dimethyl-4-oxo-2-phenyl-2,3,4,5,6,7-hexahydro-1-benzofuran-3-carboxamide

(7a). Colorless solid, yield 68\%, $193 \mathrm{mg}, \mathrm{mp} 157-158^{\circ} \mathrm{C}$; IR ( $\left.v_{\max }, \mathrm{cm}^{-1}\right): 698,761,798,1101$, 1382, 1616 (C=C), 1683 (C=O), 2916, 2949, 3188, 3365; ${ }^{1} \mathrm{H}$ NMR (400 MHz, $\left.\mathrm{CDCl}_{3}\right)$ : $\delta \mathrm{H} 1.29$ (6H, s, 2xMe), 2.43 (2H, s, H5), $2.71(2 \mathrm{H}, \mathrm{s}, \mathrm{H} 7), 3.97(1 \mathrm{H}, \mathrm{d}, J \mathrm{HH} 6.0 \mathrm{~Hz}, \mathrm{H} 3), 6.14$ (1H, d, JHH $6.0 \mathrm{~Hz}, \mathrm{H} 2), 7.34(1 \mathrm{H}, \mathrm{s}, \mathrm{NH}), 7.65-7.59(5 \mathrm{H}, \mathrm{m}$, phenyl $\mathrm{CH}), 7.68(1 \mathrm{H}, \mathrm{s}, \mathrm{NH}) ;{ }^{13} \mathrm{C}$ NMR $\left(100 \mathrm{MHz} \mathrm{CDCl}_{3}\right): \delta \mathrm{C} 28.6(\mathrm{Me}), 28.8(\mathrm{Me}), 34.5,38.3,41.2,53.2,87.9(\mathrm{C} 2), 110.3(\mathrm{C} 3 \mathrm{a})$, 126.1, 128.1 (2xCH), 129.3, 129.6, 140.8, 173.2 (C7a), $179.5(\mathrm{C}=\mathrm{O}), 197.3$ (C=O); HRMS $\left(\mathrm{ESI}^{+}\right): m / z(\mathrm{M}+\mathrm{H})^{+}$calcd for $\mathrm{C}_{17} \mathrm{H}_{20} \mathrm{NO}_{3}$ : 286.14376; found: 286.14142.

trans-4-Oxo-2-phenyl-2,3,4,5,6,7-hexahydro-1-benzofuran-3-carboxamide (7b). Colorless solid, yield 55\%, $141 \mathrm{mg}$, mp 126-128 ${ }^{\circ} \mathrm{C}$; IR ( $\left.v_{\max }, \mathrm{cm}^{-1}\right): 702,754,935,1174,1388,1614$ $(\mathrm{C}=\mathrm{C}), 1647(\mathrm{C}=\mathrm{O}), 1681(\mathrm{C}=\mathrm{O}), 2939,3186,3381 ;{ }^{1} \mathrm{H} \mathrm{NMR}\left(400 \mathrm{MHz}, \mathrm{CDCl}_{3}\right): \delta \mathrm{H}(400$ $\left.\mathrm{MHz} \mathrm{CDCl}_{3}\right) 1.94(2 \mathrm{H}, \mathrm{m}, \mathrm{H} 6), 2.28(2 \mathrm{H}, \mathrm{m}, \mathrm{H} 5), 2.42(2 \mathrm{H}, \mathrm{t}, J \mathrm{HH} 6.0 \mathrm{~Hz}, \mathrm{H} 7), 3.78(1 \mathrm{H}, \mathrm{d}$, JHH $6.0 \mathrm{~Hz}, \mathrm{H} 3), 5.34(1 \mathrm{H}, \mathrm{s}, \mathrm{NH}), 6.27(1 \mathrm{H}, \mathrm{d}, J \mathrm{HH} 6.0 \mathrm{~Hz}, \mathrm{H} 2), 7.21-7.08$ (5H, m, phenyl $\mathrm{CH}), 7.65(1 \mathrm{H}, \mathrm{s}, \mathrm{NH}) ;{ }^{13} \mathrm{C} \mathrm{NMR}\left(100 \mathrm{MHz}, \mathrm{CDCl}_{3}\right)$ : $\delta \mathrm{C} 21.4,24.3,36.6,52.9(3 \mathrm{C}), 97.1(\mathrm{C} 2)$, $111.1(\mathrm{C} 3 \mathrm{a}), 125.4(2 \mathrm{xCH}), 128.6,128.8(2 \times \mathrm{CH}), 139.9,172.1(\mathrm{C} 7 \mathrm{a}), 179.4(\mathrm{C}=\mathrm{O}), 196.7(\mathrm{C}=\mathrm{O})$; HRMS $\left(\mathrm{ESI}^{+}\right): \mathrm{m} / z(\mathrm{M}+\mathrm{Na})^{+}$calcd for $\mathrm{C}_{15} \mathrm{H}_{15} \mathrm{NO}_{3} \mathrm{Na}$ : 280.09441 ; found: 280.09520 .

trans-Ethyl 4-carbamoyl-2-methyl-5-phenyl-4,5-dihydrofuran-3-carboxylate (7c). Colorless solid, yield 50\%, $137 \mathrm{mg}$, mp 126-128 ${ }^{\circ} \mathrm{C}$; IR $\left(v_{\max }, \mathrm{cm}^{-1}\right): 694,748,1095,1263,1649(\mathrm{C}=\mathrm{C})$, 1654 (C=O), 1701 (C=O), 2920, 2987, 3199, 3375; ${ }^{1} \mathrm{H}$ NMR (400 MHz, $\left.\mathrm{CDCl}_{3}\right): \delta \mathrm{H} 1.31(3 \mathrm{H}, \mathrm{t}$, $\left.J \mathrm{HH} 7.2 \mathrm{~Hz},-\mathrm{CH}_{2} \mathrm{CH}_{3}\right), 2.34(3 \mathrm{H}, \mathrm{d}, 5 J \mathrm{HH} 1.2 \mathrm{~Hz}, \mathrm{Me}), 3.86\left(1 \mathrm{H}, \mathrm{dq},{ }^{3} J \mathrm{HH} 5.2,{ }^{5} J \mathrm{HH} 1.2 \mathrm{~Hz}\right.$, H4), $4.22\left(2 \mathrm{H}, \mathrm{q}, J \mathrm{HH} 7.2 \mathrm{~Hz},-\mathrm{OCH}_{2} \mathrm{CH}_{3}\right), 5.70(1 \mathrm{H}, \mathrm{s}, \mathrm{NH}), 6.18(1 \mathrm{H}, \mathrm{d}, J \mathrm{HH} 5.2 \mathrm{~Hz}, \mathrm{H} 5)$, $6.91(1 \mathrm{H}, \mathrm{s}, \mathrm{NH}), 7.31-7.26(2 \mathrm{H}, \mathrm{m}), 7.39-7.33(3 \mathrm{H}, \mathrm{m}) ;{ }^{13} \mathrm{C} \mathrm{NMR}\left(100 \mathrm{MHz}, \mathrm{CDCl}_{3}\right): \delta \mathrm{C} 14.3$ (Me), $15.0(\mathrm{Me}), 56.0$ (C4), 60.4170 .2 (C2), 84.7 (C5), 101.3 (C3), 125.2 (2xCH), 128.4, 128.8 $(2 \times C H), 140.4,166.5(\mathrm{C}=\mathrm{O}), 175.5(\mathrm{C}=\mathrm{O})$; HRMS $\left(\mathrm{ESI}^{+}\right): \mathrm{m} / z(\mathrm{M}+\mathrm{Na})^{+}$calcd for $\mathrm{C}_{15} \mathrm{H}_{17} \mathrm{NO}_{4} \mathrm{Na}$ : 298.10497; found: 298.10354. 
trans-4-Acetyl-5-methyl-2-phenyl-2,3-dihydrofuran-3-carboxamide (7d). Colorless solid, yield 41\%, $100 \mathrm{mg}, \mathrm{mp} 125-128{ }^{\circ} \mathrm{C}$; IR $\left(v_{\max }, \mathrm{cm}^{-1}\right): 692,748,941,1261,1581(\mathrm{C}=\mathrm{C}), 1660$ $(\mathrm{C}=\mathrm{O}), 1676(\mathrm{C}=\mathrm{O}), 2848,2914,3186,3348 ;{ }^{1} \mathrm{H}$ NMR (400 MHz, $\left.\mathrm{CDCl}_{3}\right): \delta \mathrm{H} 2.53(3 \mathrm{H}, \mathrm{s}, \mathrm{Me})$, $2.56(3 \mathrm{H}, \mathrm{s}, \mathrm{Me}), 4.15(1 \mathrm{H}, \mathrm{d}, J \mathrm{HH} 4.81 \mathrm{~Hz}, \mathrm{H} 3), 5.80(1 \mathrm{H}, \mathrm{s}, \mathrm{NH}), 6.38(1 \mathrm{H}, \mathrm{d}, J \mathrm{HH} 4.83 \mathrm{~Hz}$, $\mathrm{H} 2), 7.22(1 \mathrm{H}, \mathrm{s}, \mathrm{NH}), 7.43(2 \mathrm{H}, \mathrm{d}, J \mathrm{HH} 6.11 \mathrm{~Hz}$, phenyl $\mathrm{CH}), 7.49(3 \mathrm{H}, \mathrm{m}$, phenyl $\mathrm{CH}) ;{ }^{13} \mathrm{C}$ NMR (100 MHz, $\left.\mathrm{CDCl}_{3}\right)$ : $\delta \mathrm{C} 16.2(\mathrm{Me}), 29.3(\mathrm{Me}), 55.8(3 \mathrm{C}), 84.4$ (2C), 114.0 (4C), 125.2 $(2 \times C H), 128.5(2 \times C H), 128.8,140.1,169.9(5 \mathrm{C}), 173.2(\mathrm{C}=\mathrm{O}), 194.4(\mathrm{C}=\mathrm{O}) ; \mathrm{HRMS}\left(\mathrm{ESI}^{+}\right): \mathrm{m} / z$ $(\mathrm{M}+\mathrm{H})^{+}$calcd for $\mathrm{C}_{14} \mathrm{H}_{16} \mathrm{NO}_{3}$ : 246.11246; found: 246.11111 .

trans-6,6-Dimethyl-4-oxo-2-(thiophen-2-yl)-2,3,4,5,6,7-hexahydro-1-benzofuran-3-

carboxamide (7e). Colorless solid, yield 55\%, $160 \mathrm{mg}, \mathrm{mp} 153-155^{\circ} \mathrm{C}$; IR ( $\left.v_{\max }, \mathrm{cm}^{-1}\right)$ : 613, 640, 713, 800, 1060, 1101, 1379, $1612(\mathrm{C}=\mathrm{C}), 1685(\mathrm{C}=\mathrm{O}), 2924,2952,3184,3315 ;{ }^{1} \mathrm{H}$ NMR (400 MHz, CDCl 3 ): $\delta \mathrm{H} 1.14(3 \mathrm{H}, \mathrm{s}, \mathrm{Me}), 1.15(3 \mathrm{H}, \mathrm{s}, \mathrm{Me}), 2.34(2 \mathrm{H}, \mathrm{s}, \mathrm{H} 5), 2.40$ (2H, d, JHH $2.0 \mathrm{~Hz}, \mathrm{H} 7), 4.11(1 \mathrm{H}, \mathrm{d}, J \mathrm{HH} 5.60 \mathrm{~Hz}, \mathrm{H} 3), 5.39(1 \mathrm{H}, \mathrm{s}, \mathrm{NH}), 6.68(1 \mathrm{H}, \mathrm{d}, J \mathrm{HH} 5.60 \mathrm{~Hz}, \mathrm{H} 2)$, $6.99(1 \mathrm{H}, \mathrm{dd}, J \mathrm{HH} 5.20,3.60 \mathrm{~Hz}$, thiophene $\mathrm{CH}), 7.11(1 \mathrm{H}, \mathrm{d}, J \mathrm{HH} 3.2 \mathrm{~Hz}$, thiophene $\mathrm{CH}), 7.30$ $(1 \mathrm{H}, \mathrm{d}, J \mathrm{HH} 4.8 \mathrm{~Hz}$, thiophene $\mathrm{CH}), 7.75(1 \mathrm{H}, \mathrm{s}, \mathrm{NH}) ;{ }^{13} \mathrm{C} \mathrm{NMR}\left(100 \mathrm{MHz}, \mathrm{CDCl}_{3}\right): \delta \mathrm{C} 28.7$ (2xMe), 34.6, 38.3, 51.2, 52.9 (C3), 83.9 (C2), 109.8 (C3a), 126.3, 126.6, 127.3, 142.5, 171.7 (C7a), $177.9(\mathrm{C}=\mathrm{O}), 196.3(\mathrm{C}=\mathrm{O})$; HRMS $\left(\mathrm{ESI}^{+}\right): \mathrm{m} / \mathrm{z}(\mathrm{M}+\mathrm{Na})^{+}$calcd for $\mathrm{C}_{15} \mathrm{H}_{17} \mathrm{NO}_{3} \mathrm{SNa}$ : 314.08213; found: 314.08170 .

trans-4-Oxo-2-(thiophen-2-yl)-2,3,4,5,6,7-hexahydro-1-benzofuran-3-carboxamide

(7f). Colorless solid, yield 54\%, $142 \mathrm{mg}, \mathrm{mp} \mathrm{100-103}{ }^{\circ} \mathrm{C}$; IR ( $\left.v_{\max }, \mathrm{cm}^{-1}\right): 705,756,837,904,1377$, 1400, 1608 (C=C), $1670(\mathrm{C}=\mathrm{O}), 1697(\mathrm{C}=\mathrm{O}), 2902,2947,3192,3315 ;{ }^{1} \mathrm{H}$ NMR (400 MHz, $\left.\mathrm{CDCl}_{3}\right): \delta \mathrm{H} 2.09$ (2H, m, H6), $2.45(2 \mathrm{H}, \mathrm{m}, \mathrm{H} 5), 2.53(2 \mathrm{H}, \mathrm{t}, J \mathrm{HH} 6.0 \mathrm{~Hz}, \mathrm{H} 7), 4.10(1 \mathrm{H}, \mathrm{d}, J \mathrm{HH}$ $5.2 \mathrm{~Hz}, \mathrm{H} 3), 5.80(1 \mathrm{H}, \mathrm{s}, \mathrm{NH}), 6.65(1 \mathrm{H}, \mathrm{d}, J \mathrm{HH} 5.60 \mathrm{~Hz}, \mathrm{H} 2), 6.99(1 \mathrm{H}, \mathrm{dd}, J \mathrm{HH} 4.8,3.6 \mathrm{~Hz}$, thiophene $\mathrm{CH}), 7.11(1 \mathrm{H}, \mathrm{d}, J \mathrm{HH} 3.2 \mathrm{~Hz}$, thiophene $\mathrm{CH}), 7.31(1 \mathrm{H}, \mathrm{dd}, J \mathrm{HH} 4.8,1.2 \mathrm{~Hz}$, thiophene $\mathrm{CH}), 7.76(1 \mathrm{H}, \mathrm{s}, \mathrm{NH}) ;{ }^{13} \mathrm{C} \mathrm{NMR}\left(100 \mathrm{MHz}, \mathrm{CDCl}_{3}\right): \delta \mathrm{C} 21.6,24.5,36.7,53.0(\mathrm{C} 3)$, 83.6 (C2), 111.4 (C3a), 126.3, 126.6, 127.3, 142.3, 171.9 (C7a), $178.7(\mathrm{C}=\mathrm{O}), 196.9(\mathrm{C}=\mathrm{O})$; HRMS $\left(\mathrm{ESI}^{+}\right): \mathrm{m} / \mathrm{z}(\mathrm{M}+\mathrm{Na})^{+}$calcd for $\mathrm{C}_{13} \mathrm{H}_{13} \mathrm{NO}_{3} \mathrm{SNa}$ : 286.05083; found: 286.05155 .

trans-Ethyl 4-carbamoyl-2-methyl-5-(thiophen-2-yl)-4,5-dihydrofuran-3-carboxylate (7g). Colorless solid, yield 46\%, $129 \mathrm{mg}, \mathrm{mp} \mathrm{82-86}{ }^{\circ} \mathrm{C}$; IR $\left(v_{\max }, \mathrm{cm}^{-1}\right): 696,765,975,1095,1201$, $1649(\mathrm{C}=\mathrm{C}), 1658(\mathrm{C}=\mathrm{O}), 1701(\mathrm{C}=\mathrm{O}), 2920,2981,3197,3379 ;{ }^{1} \mathrm{H}$ NMR (400 MHz, $\left.\mathrm{CDCl}_{3}\right)$ : $\delta \mathrm{H} 1.36(3 \mathrm{H}, \mathrm{t}, J \mathrm{HH} 7.12 \mathrm{~Hz}, \mathrm{Me}), 2.30(3 \mathrm{H}, \mathrm{d}, J \mathrm{HH} 1.20 \mathrm{~Hz}, \mathrm{Me}), 4.04(1 \mathrm{H}, \mathrm{dd}, J \mathrm{HH} 4.62,1.24$ Hz, H3), 4.27 (2H, q, JHH $\left.7.13 \mathrm{~Hz},-\mathrm{OCH}_{2}-\right), 5.35(1 \mathrm{H}, \mathrm{s}, \mathrm{NH}), 6.46(1 \mathrm{H}, \mathrm{d}, J \mathrm{HH} 4.65 \mathrm{~Hz}, \mathrm{H} 2)$, $7.00(1 \mathrm{H}, \mathrm{dd}, J \mathrm{HH} 5.03,3.56 \mathrm{~Hz}$, thiophene $\mathrm{CH}), 7.10(1 \mathrm{H}, \mathrm{d}, J \mathrm{HH} 3.52 \mathrm{~Hz}$, thiophene $\mathrm{CH})$, $7.29(1 \mathrm{H}, \mathrm{s}, \mathrm{NH}), 7.31(1 \mathrm{H}, \mathrm{dd}, \mathrm{JHH} 5.07,1.12 \mathrm{~Hz}$, thiophene $\mathrm{CH}) ;{ }^{13} \mathrm{C} \mathrm{NMR}(100 \mathrm{MHz}$, $\left.\mathrm{CDCl}_{3}\right): \delta \mathrm{C} 14.5(\mathrm{Me}), 15.4(\mathrm{Me}), 55.9,60.8(\mathrm{C} 4), 81.0(\mathrm{C} 5), 101.7(\mathrm{C} 3), 125.7,126.1,127.1$, 143.0, $166.7(\mathrm{C} 2), 169.7(\mathrm{C}=\mathrm{O}), 172.9(\mathrm{C}=\mathrm{O})$; HRMS $\left(\mathrm{ESI}^{+}\right): \mathrm{m} / \mathrm{z}(\mathrm{M}+\mathrm{Na})^{+}$calcd for $\mathrm{C}_{13} \mathrm{H}_{15} \mathrm{NO}_{4} \mathrm{SNa}: 304.06139$; found: 304.06149 .

trans-4-Acetyl-5-methyl-2-(thiophen-2-yl)-2,3-dihydrofuran-3-carboxamide (7h). Yellow oil, yield 52\%, $130 \mathrm{mg}$; IR $\left(v_{\max }, \mathrm{cm}^{-1}\right): 704,750,935,827,1207,1384,1578(\mathrm{C}=\mathrm{C}), 1670$ $(\mathrm{C}=\mathrm{O}), 2914,3006,3190,3415 ;{ }^{1} \mathrm{H}$ NMR (400 MHz, $\left.\mathrm{CDCl}_{3}\right)$ : $\delta \mathrm{H} 2.36(3 \mathrm{H}, \mathrm{s}, \mathrm{Me}), 2.43$ (3H, s, 
Me), 4.16 (1H, d, J HH 4.00 Hz, H3), 5.64 (1H, s, NH), 6.47 (1H, d, JHH 4.10 Hz, H2), 7.00 $(1 \mathrm{H}, \mathrm{t}, J \mathrm{HH} 4.52 \mathrm{~Hz}$, thiophene $\mathrm{CH}), 7.08(1 \mathrm{H}, \mathrm{d}, J \mathrm{HH} 3.15 \mathrm{~Hz}$, thiophene $\mathrm{CH}), 7.15(1 \mathrm{H}, \mathrm{s}$, $\mathrm{NH}), 7.30(1 \mathrm{H}, \mathrm{d}, J 5.09 \mathrm{~Hz}$, thiophene $\mathrm{CH}) ;{ }^{13} \mathrm{C} \mathrm{NMR}\left(100 \mathrm{MHz}, \mathrm{CDCl}_{3}\right): \delta \mathrm{C} 16.5(\mathrm{Me})$,

29.5 (Me), 55.7 (C3), 80.8 (C2), 114.0 (C4), 125.9, 126.2, 127.1, 142.5, 169.5 (C5), 172.9 $(\mathrm{C}=\mathrm{O}), 194.6(\mathrm{C}=\mathrm{O})$; HRMS $\left(\mathrm{ESI}^{+}\right): \mathrm{m} / z(\mathrm{M}+\mathrm{H})^{+}$calcd for $\mathrm{C}_{12} \mathrm{H}_{14} \mathrm{NO}_{3} \mathrm{~S}: 252.06889$; found: 252.06908 .

trans-6,6-Dimethyl-2-(5-methylfuran-2-yl)-4-oxo-2,3,4,5,6,7-hexahydro-1-benzofuran-3carboxamide (7i). Yellow solid, yield 80\%, $231 \mathrm{mg}, \mathrm{mp} 143-145{ }^{\circ} \mathrm{C}$; IR $\left(v_{\max }, \mathrm{cm}^{-1}\right): 621,665$, 781, 879, 1020, 1070, 1251, 1388, 1591 (C=C), $1614(\mathrm{C}=\mathrm{O}), 1683(\mathrm{C}=\mathrm{O}), 2920,2964,3175$, 3383; ${ }^{1} \mathrm{H}$ NMR (400 MHz, $\left.\mathrm{CDCl}_{3}\right): \delta \mathrm{H} 1.11(6 \mathrm{H}, \mathrm{s}, \mathrm{Me}), 2.26(3 \mathrm{H}, \mathrm{s}, \mathrm{Me}), 2.32(2 \mathrm{H}, \mathrm{d}, J \mathrm{HH}$ $1.60 \mathrm{~Hz}, \mathrm{H} 5), 2.35$ (2H, d, JHH 1.20 Hz, H7), 4.25 (1H, d, JHH 6.0 Hz, H3), 5.53 (1H, s, NH), $5.94(1 \mathrm{H}, \mathrm{dq}, J \mathrm{HH} 3.60,0.40 \mathrm{~Hz}$, furan $\mathrm{CH}), 6.34(1 \mathrm{H}, \mathrm{d}, J \mathrm{HH} 3.2 \mathrm{~Hz}$, furan $\mathrm{CH}), 6.35(1 \mathrm{H}$, d, JHH $6.0 \mathrm{~Hz}, \mathrm{H} 2), 7.84(1 \mathrm{H}, \mathrm{s}, \mathrm{NH}) ;{ }^{13} \mathrm{C} \mathrm{NMR}\left(100 \mathrm{MHz}, \mathrm{CDCl}_{3}\right)$ : $\delta \mathrm{C} 13.9,28.6,28.8,34.5$, 38.3, 48.7, 51.1 (C3), 81.3 (C2), 106.9, 110.3, 111.0, 149.2, 154.1, 171.9 (C7a), 178.2 (C=O), $196.2(\mathrm{C}=\mathrm{O})$; HRMS $\left(\mathrm{ESI}^{+}\right): \mathrm{m} / z(\mathrm{M}+\mathrm{H})^{+}$calcd for $\mathrm{C}_{16} \mathrm{H}_{20} \mathrm{NO}_{4}$ : 290.13868; found: 290.13923 .

Ethyl trans-3-carbamoyl-5,5'-dimethyl-2,3-dihydro-[2,2'-bifuran]-4-carboxylate (7j). Yellow solid, yield 67\%, $186 \mathrm{mg}, \mathrm{mp} 133-135^{\circ} \mathrm{C}$; IR $\left(v_{\max }, \mathrm{cm}^{-1}\right): 653,796,962,1124,1217$, 1319, 1381, $1635(\mathrm{C}=\mathrm{C}), 1654(\mathrm{C}=\mathrm{O}), 1678(\mathrm{C}=\mathrm{O}), 2918,2985,3165,3352 ;{ }^{1} \mathrm{H}$ NMR (400 $\left.\mathrm{MHz}_{\mathrm{CDCl}}\right): \delta \mathrm{H} 1.32(3 \mathrm{H}, \mathrm{t}, J \mathrm{HH} 7.20 \mathrm{~Hz}, \mathrm{Me}), 2.23(3 \mathrm{H}, \mathrm{s}, \mathrm{Me}), 2.27(3 \mathrm{H}, \mathrm{s}, 5.84(1 \mathrm{H}, \mathrm{s}$, $\mathrm{NH}), \mathrm{Me}), 4.14\left(1 \mathrm{H}, \mathrm{d}, J \mathrm{HH} 5.60 \mathrm{~Hz}, \mathrm{H} 3\right.$ ) $, 4.23\left(2 \mathrm{H}, \mathrm{q}, J \mathrm{HH} 7.20 \mathrm{~Hz},-\mathrm{OCH}_{2}-\right), 5.92(1 \mathrm{H}$, d, JHH $3.20 \mathrm{~Hz}$, furan CH), $6.08\left(1 \mathrm{H}, \mathrm{d}, J \mathrm{HH} 5.60 \mathrm{~Hz}, \mathrm{H} 2^{\prime}\right), 6.28(1 \mathrm{H}, \mathrm{d}, J \mathrm{HH} 3.20 \mathrm{~Hz}$, furan CH), $6.95(1 \mathrm{H}, \mathrm{s}, \mathrm{NH}) ;{ }^{13} \mathrm{C} \mathrm{NMR}\left(100 \mathrm{MHz}, \mathrm{CDCl}_{3}\right)$ : $\delta \mathrm{C} 13.9(\mathrm{Me}), 14.6(\mathrm{Me}), 15.3(\mathrm{Me}), 52.1,60.6$, 78.6, 102.0, 106.7, 110.4, 149.8, 153.7, $166.8(\mathrm{C}=\mathrm{O}), 169.8\left(\mathrm{C}^{\prime}\right), 173.3(\mathrm{C}=\mathrm{O})$; HRMS (ESI $\left.{ }^{+}\right)$: $\mathrm{m} / \mathrm{z}(\mathrm{M}+\mathrm{Na})^{+}$calcd for $\mathrm{C}_{14} \mathrm{H}_{17} \mathrm{NO}_{5} \mathrm{Na}: 302.09989$; found: 302.10051 .

trans-4-Acetyl-5,5'-dimethyl-2,3-dihydro-[2,2'-bifuran]-3-carboxamide (7k). Pale yellow solid, yield 70\%, $174 \mathrm{mg}$, mp 150-152 ${ }^{\circ} \mathrm{C}$; IR $\left(v_{\max }, \mathrm{cm}^{-1}\right): 663,796,900,943,1213,1392,1595$ $(\mathrm{C}=\mathrm{C}), 1672(\mathrm{C}=\mathrm{O}), 1681(\mathrm{C}=\mathrm{O}), 2848,2918,3153,3327 ;{ }^{1} \mathrm{H} \mathrm{NMR}\left(400 \mathrm{MHz}, \mathrm{CDCl}_{3}\right): \delta \mathrm{H}$ $2.26(3 \mathrm{H}, \mathrm{s}, \mathrm{Me}), 2.29(3 \mathrm{H}, \mathrm{s}, \mathrm{Me}), 2.37(3 \mathrm{H}, \mathrm{d}, J \mathrm{HH} 0.8 \mathrm{~Hz}, \mathrm{Me}), 4.24(1 \mathrm{H}, \mathrm{d}, J \mathrm{HH} 5.2 \mathrm{~Hz}$, H3),5.70 (1H, s, NH), $5.91(1 \mathrm{H}, \mathrm{dq}, J \mathrm{HH} 3.2,0.8 \mathrm{~Hz}$, furan $\mathrm{CH}), 6.11(1 \mathrm{H}, \mathrm{d}, J \mathrm{HH} 5.2 \mathrm{~Hz}$, $\left.\mathrm{H} 2^{\prime}\right), 6.26(1 \mathrm{H}, \mathrm{d}, J \mathrm{HH} 3.2 \mathrm{~Hz}$, furan $\mathrm{CH}), 7.14(1 \mathrm{H}, \mathrm{s}, \mathrm{NH}) ;{ }^{13} \mathrm{C} \mathrm{NMR}\left(100 \mathrm{MHz}, \mathrm{CDCl}_{3}\right): \delta \mathrm{C}$ $13.9(\mathrm{Me}), 16.5(\mathrm{Me}), 29.6(\mathrm{Me}), 52.0,78.4,106.7,110.5,114.7,149.6,153.8,169.9$ (C5'), $173.1(\mathrm{C}=\mathrm{O}), 194.5(\mathrm{C}=\mathrm{O})$; HRMS $\left(\mathrm{ESI}^{+}\right): \mathrm{m} / z(\mathrm{M}+\mathrm{Na})^{+}$calcd for $\mathrm{C}_{13} \mathrm{H}_{15} \mathrm{NO}_{4} \mathrm{Na}$ : 272.08932; found: 272.08976 .

trans-6,6-Dimethyl-4-oxo-2-(thiophen-3-yl)-2,3,4,5,6,7-hexahydrobenzofuran-3-

carboxamide (7l). Colorless solid, yield $36 \%, 104 \mathrm{mg}, \mathrm{mp} 148-150{ }^{\circ} \mathrm{C}$; IR ( $\left.v_{\max }, \mathrm{cm}^{-1}\right)$ : 700, 965, 1095, 1212, $1618(\mathrm{C}=\mathrm{C}), 1675(\mathrm{C}=\mathrm{O}), 1703(\mathrm{C}=\mathrm{O}), 2920,2983,3194,3362 ;{ }^{1} \mathrm{H}$ NMR (400 $\left.\mathrm{MHz}_{\mathrm{CDCl}}\right)$ : $\delta \mathrm{H} 1.14(6 \mathrm{H}, \mathrm{s}), 2.32(2 \mathrm{H}, \mathrm{s}), 2.42(2 \mathrm{H}, \mathrm{s}), 4.00(1 \mathrm{H}, \mathrm{d}, J \mathrm{HH} 6.0 \mathrm{~Hz}, \mathrm{H} 3), 5.94$ $(1 \mathrm{H}, \mathrm{s}, \mathrm{NH}), 6.50(1 \mathrm{H}, \mathrm{d}, J \mathrm{HH} 6.0 \mathrm{~Hz}, \mathrm{H} 2), 7.01(1 \mathrm{H}, \mathrm{dd}, J \mathrm{HH} 4.8,0.8 \mathrm{~Hz}$, thiophene $\mathrm{CH}), 7.28$ $(1 \mathrm{H}, \mathrm{d}, J \mathrm{HH} 7.2 \mathrm{~Hz}$, thiophene $\mathrm{CH}), 7.35(1 \mathrm{H}, \mathrm{dd}, J \mathrm{HH} 5.2,4.8 \mathrm{~Hz}$, thiophene $\mathrm{CH}), 7.80(1 \mathrm{H}, \mathrm{s}$, $\mathrm{NH}) ;{ }^{13} \mathrm{C}$ NMR $\left(100 \mathrm{MHz}, \mathrm{CDCl}_{3}\right): \delta \mathrm{C} 28.3,28.6,34.2,38.1,50.9,51.8,84.1,109.6,122.2$, 
124.9, 127.3, 140.6, $171.9(\mathrm{C}=\mathrm{O}), 178.1(\mathrm{C} 7 \mathrm{a}), 196.0(\mathrm{C}=\mathrm{O})$; HRMS $\left(\mathrm{ESI}^{+}\right): \mathrm{m} / z(\mathrm{M}+\mathrm{Na})^{+}$calcd for $\mathrm{C}_{15} \mathrm{H}_{17} \mathrm{NO}_{3} \mathrm{SNa}$ : 314.08213; found: 314.08881 .

\section{Acknowledgements}

The authors are grateful to the Kocaeli University (BAP 2010/57 and 2012/28) Science Research Foundations for financial support. Authors thank to Yunus Zorlu (GYTE) for X-ray crystallography analyses. A. Ustalar thanks to TUBITAK for doctoral fellowship.

\section{Supplementary Material}

Copies of NMR spectra for all compounds, X-ray crystal data for compounds $7 \mathbf{b}, \mathbf{7 i}$ and $\mathbf{7 j}$ (CCDC nos. 997073, 997070 and 997071, respectively). Supplementary data and crystallographic data associated with this article can be found in the online version, at http://dx.doi.org/

\section{References}

1. Matsuya, Y.; Suzuki, N.; Kobayashi, S. Y.; Miyahara, T.; Ochiai, H.; Nemoto, H. Bioorg. Med. Chem. 2010, 18, 1477-1481.

http://dx.doi.org/10.1016/j.bmc.2010.01.014

2. Freire, C. P. V.; Ferreira, S. B.; de Oliveira, N. S. M.; Matsuura, A. B. J.; Gama, I. L.; da Silva, F. C.; de Souza, M. C. B. V.; Lima, E. S.; Ferreira, V. F. Med. Chem. Commun. 2010, 1, 229-232. http://dx.doi.org/10.1039/C0MD00074D

3. Koike, T.; Takai, T.; Hoashi, Y.; Nakayama, M.; Kosugi, Y.; Nakashima, M.; Yoshikubo, S.; Hirai, K.; Uchikawa, O. J. Med. Chem. 2011, 54, 4207-4218. http://dx.doi.org/10.1021/jm200385u

4. Elbe, H.-L.; Kunisch, F.; Bielefeldt, D.; Tiemann, R.; Stenzel, K.; Dutzmann, S.; Kugler, M.; Schrage, H. U.S. Patent 6107 336, 2000.

5. Logoglu, E.; Yilmaz, M.; Katircioglu, H.; Yakut, M.; Mercan, S. Med. Chem. Res. 2010, 19, 490-487. http://dx.doi.org/10.1007/s00044-009-9206-8

6. Mellor, J. M.; Mohammed, S. Tetrahedron 1993, 49, 7547-7556. http://dx.doi.org/10.1016/S0040-4020(01)87229-X

7. Garzino, F.; Méou, A.; Brun, P. Tetrahedron Lett. 2000, 41, 9803-9807. http://dx.doi.org/10.1016/S0040-4039(00)01729-9

8. Snider, B. B. Chem. Rev. 1996, 96, 339-363. 
http://dx.doi.org/10.1021/cr950026m

9. Wang, G.-W.; Dong, Y.-W.; Wu, P.; Yuan, T.-T.; Shen, Y.-B. J. Org. Chem. 2008, 73, 70887095.

http://dx.doi.org/10.1021/jo800870z

10. Deliomeroglu, M. K.; Deniz, C.; Caliskan, R.; Balci, M. Tetrahedron 2012, 68, 5838-5844. http://dx.doi.org/10.1016/j.tet.2012.05.003

11. Nair, V.; Mathew, J.; Radhakrishnan, K. V. J. Chem. Soc., Perkin Trans. 1, 1996, 1487-1492. http://dx.doi.org/10.1039/P19960001487

12. Kobayashi, K.; Sakashita, K.; Akamatsu, H.; Tanaka, K.; Uchida, M.; Uneda, T.; Kitamura, T.; Morikawa, O.; Konishi, H. Heterocycles 1999, 51, 2881-2892.

http://dx.doi.org/10.3987/COM-99-8688

13. Nair, V.; Deepthi, A. Chem. Rev. C 2007, 107, 1862-1891.

http://dx.doi.org/10.1021/cr068408n

14. S. C. Roy, P. K. Mandal, Tetrahedron 1996, 52, 2193-2198.

http://dx.doi.org/10.1016/0040-4020(95)01050-5

15. Shanmugam, P.; Kumar, K. H.; Perumal, P. T. J. Het. Chem., 2007, 44, 827-830. http://dx.doi.org/10.1002/jhet.5570440412

16. Zhang, W.; Huo, C. D.; Liu, Z. G.; Liu, Z. L. Chin. Chem. Lett., 2004, 15, 389-391. http://ir.lzu.edu.cn/handle/262010/79583

17. Lee, Y. R.; Kim, B. S.; Kweon, H. I. Tetrahedron, 2000, 56, 3867-3874. http://dx.doi.org/10.1016/S0040-4020(00)00307-0

18. Yilmaz, M.; Uzunalioglu, N.; Pekel, A. T. Tetrahedron 2005, 61, 8860-8867. http://dx.doi.org/10.1016/j.tet.2005.07.019

19. Burgaz, E. V.; Yilmaz, M.; Pekel, A. T.; Oktemer, A. Tetrahedron 2007, 63, 7229-7239. http://dx.doi.org/10.1016/j.tet.2007.04.088

20. Y1lmaz, M.; Uzunalioglu, N.; Yakut, M.; Pekel, A. T. Turk. J. Chem. 2008, 32, 411-422. http://journals.tubitak.gov.tr/chem/issues/kim-08-32-4/kim-32-4-2-0708-25.pdf

21. Yilmaz, M. Tetrahedron 2011, 67, 8255-8263. http://dx.doi.org/10.1016/j.tet.2011.08.098

22. Bicer, E.; Yilmaz, M; Karatas, M.; Pekel, A. T. Helv. Chim. Acta 2012, 95, 795-804. http://dx.doi.org/10.1002/hlca.201100397

23. Bicer, E.; Yilmaz, M.; Burgaz, E. V.; Pekel, A. T. Helv. Chim. Acta 2013, 96, 135-141. http://dx.doi.org/10.1002/hlca.201200098

24. Yilmaz, M.; Bicer, E.; Ustalar, A.; Pekel A.T. Arkivoc 2014 (v) 225-236. http://dx.doi.org/10.3998/ark.5550190.0015.500 http://www.arkat-usa.org/get-file/51123/

25. Yilmaz, M.; Burgaz, E. V.; Yakut, M.; Bicer, E. J. Chin. Chem. Soc. 2014, 61, 1101-1107. http://dx.doi.org/10.1002/jccs.201400173

26. Alagoz, O.; Yilmaz, M.; Pekel, A.T.; Graiff, C.; Maggi, R. RSC Adv. 2014, 4 14644-14654. http://dx.doi.org/10.1039/c3ra48015a

27. Burgaz, E. V.; Yilmaz, M.; Pekel, A. T.; Oktemer, A. Tetrahedron, 2015, 71, 4888. 
http://dx.doi.org/10.1016/j.tet.2015.05.077

28. Drauz, K.; Kleemann, A.; Wolf-Heuss, E. U.S. Patent 47287431988. 
29. Yu, H.; Han, J.; Chen, J.; Deng, H.; Shao, M.; Zhang, H.; Cao W. Eur. J. Org. Chem. 2012, 16, 3142-3150.

http://dx.doi.org/10.1002/ejoc.201200180

30. S. Martinet, A. Méou, P. Brun, Magn. Reson. Chem., 2007, 45, 182-184.

http://dx.doi.org/10.1002/mrc.1933 